MAD/TH-92-03

hep-th/9207093

June 1992

\title{
Collective Coordinate Action for Charged Sigma-Model Vortices in Finite Geometries
}

\author{
Theodore J. Allen* \\ Department of Physics \\ University of Wisconsin, Madison, WI 53706 USA
}

\begin{abstract}
In this Letter the method of Lund is applied to formulate a variational principle for the motion of charged vortices in an effective non-linear Schrödinger field theory describing finite size two-dimensional quantum Hall samples under the influence of an arbitrary perpendicular magnetic field. Freezing out variations in the modulus of the effective field yields a $U(1)$ sigma-model. A duality transformation on the sigma-model reduces the problem to finding the Green function for a related electrostatics problem. This duality illuminates the plasma analogy to the Laughlin wave function.
\end{abstract}

* tjallen@wishep.physics.wisc.edu 
The fractional quantum Hall effect shares a number of remarkable features with the macroscopic quantum phenomena of superconductivity and superfluidity. It occurs only at very low temperatures, is dissipationless and is a result of a broken (translational) symmetry. ${ }^{[1]}$ It is reasonable to assume that there should exist an effective field theory ${ }^{[2]}$ for the macroscopic quantum state of fractional quantum Hall systems. As in the effective theories of the other macroscopic quantum phenomena, there are vortex excitations in the effective theory of the quantum Hall effect. However, unlike their better-known cousins, these vortices are point-like (because of the two-dimensional nature of the effect) and play the central role in the physics of this phenomenon.

An effective field theory, or Ginzburg-Landau, description of the fractional quantum Hall effect provides a beautiful characterization of the fractionally charged quasiparticles necessary for fractional conductivity as charged vortex excitations of the quantum Hall fluid. The quantum mechanics of a charged vortex yields the lowest Landau level states of a particle of equal charge, ${ }^{[3]}$ even though vortex mechanics is quite different in detail from the usual charged particle mechanics. Because vortices are extended field configurations, the geometry of the sample in which they occur determines the Hamiltonian and hence their dynamics. The purpose of this Letter is to derive the action for arbitrary sample geometry.

Vortices in an infinite plane have a pair-wise logarithmic interaction as well as a singlevortex interaction with the background magnetic field. When boundaries are present, there is an additional vortex-boundary interaction which can be written with the help of image vortices in simple geometries.

The logarithmic interaction is analogous to vortex-vortex interactions in fluid mechanics. The center of one vortex is carried along with the bulk motion of fluid around another. The external vector potential corresponds to a background motion of the fluid with vorticity density equal to the applied magnetic field. Motion in a uniform field, for instance, is analogous to a uniform rotation about some point. In this Letter, the duality between this viewpoint and an electrostatic description will be used to derive the expression for the collective Hamiltonian in a two-dimensional bounded region for an arbitrary applied magnetic field.

The derivation of the collective action for the motion of the vortex centers starts with the elegant idea of F. Lund, ${ }^{[4]}$ which is to substitute the collective vortex ansatz for the field configuration with vortices at $\mathbf{X}^{\mathrm{A}}, \mathrm{A}=1, \ldots, N$, into the effective field theory action and 
reduce the action to a function of the collective coordinates,

$$
S_{V}\left[\left\{\mathbf{X}^{\mathrm{A}}(t)\right\}\right]=S_{\mathrm{EFT}}\left[\psi\left(\mathbf{x}, t ;\left\{\mathbf{X}^{\mathrm{A}}(t)\right\}\right)\right]
$$

Here we follow Lund's method but we must be careful in choosing the multi-vortex ansatz, $\psi=\psi\left(\mathbf{x}, t ;\left\{\mathbf{X}^{\mathrm{A}}(t)\right\}\right)$. The usual ansatz in an infinite region,

$$
\psi=\sqrt{\rho_{0}} \exp \left\{i \sum_{\mathrm{A}} n_{\mathrm{A}} \tan ^{-1}\left(\frac{x_{2}-X_{2}^{\mathrm{A}}(t)}{x_{1}-X_{1}^{\mathrm{A}}(t)}\right)\right\}
$$

with $\rho_{0}$ constant is not valid because it violates the boundary conditions.

The correct boundary conditions on the ansatz follow from considering variations on the field $\psi$ which are arbitrary at the boundary. Thus, along with the usual Euler-Lagrange equations of motion in the region $\Sigma$, one must impose the Ginzburg-Landau conditions

$$
\left.\hat{\mathbf{n}} \cdot \frac{\partial L}{\partial(\nabla \bar{\psi})}\right|_{\partial \Sigma}=0
$$

at a free boundary. In a fluid mechanical system this would enforce the requirement that the normal velocity of the fluid vanish at the walls of a container. In a quantum mechanical system the boundary conditions force the vanishing of the normal electric current.

In Ref. [3] the non-linear Schrödinger Lagrangian,

$$
\mathcal{L}=i \bar{\psi} \dot{\psi}-\frac{\alpha}{|\psi|^{2}}|(\nabla-i e \mathbf{A}) \psi|^{2}-V\left(|\psi|^{2}\right)
$$

for a charged field $\psi$ in a background magnetic field, $B(\mathbf{x})=\nabla \times \mathbf{A}$, was proposed as an effective theory for the fractional quantum Hall effect which has charged vortex solutions. The equations of motion for this Lagrangian are solved by configurations of the effective field

$$
\psi=\sqrt{\rho_{0}} \exp (i \phi)
$$

with constant $\rho_{0}$, and harmonic phase,

$$
\nabla^{2} \phi=0
$$

in a Coulomb gauge, $\nabla \cdot \mathbf{A}=0$. 
Because (6) is linear, single vortex excitations can be superposed to form multi-vortex excitations, $\phi=\sum_{\mathrm{A}} n_{\mathrm{A}} \phi_{\mathrm{A}}$. The $\phi_{\mathrm{A}}$ are unit vortex solutions and the integers $n_{\mathrm{A}}$ are the strengths of the vortices centered at $\mathrm{X}^{\mathrm{A}}$. The condition characterizing a unit vortex with center $\mathbf{X}^{\mathrm{A}}$ is

$$
\nabla \times \nabla \phi_{\mathrm{A}}(\mathbf{x})=2 \pi \delta^{(2)}\left(\mathbf{x}-\mathbf{X}^{\mathrm{A}}\right) .
$$

Eqs. (6) and (7) are much like the Bianchi identity and equations of motion for a field strength $\nabla \phi$. It is well known that one can dualize such equations by using a volume form. In this case we shall use the area form $\varepsilon=\left(\begin{array}{cc}0 & 1 \\ -1 & 0\end{array}\right)$ to define a dual potential $\chi$,

$$
\nabla \phi_{\mathrm{A}}=\varepsilon \cdot \nabla \chi_{\mathrm{A}} .
$$

The equations (6) and (7) for $\phi$ become

$$
\begin{aligned}
\nabla^{2} \chi_{\mathrm{A}}(\mathbf{x}) & =-2 \pi \delta^{(2)}\left(\mathbf{x}-\mathbf{X}^{\mathrm{A}}\right), \\
\nabla \times \nabla \chi_{\mathrm{A}} & =0 .
\end{aligned}
$$

It is useful to dualize the gauge and field-strength equations for $\mathbf{A}$ as well,

$$
\begin{aligned}
\mathbf{A} & =\varepsilon \cdot \nabla \Upsilon, \\
\nabla \times \nabla \Upsilon & =0, \\
\nabla^{2} \Upsilon & =-B(\mathbf{x}) .
\end{aligned}
$$

The boundary conditions on $\phi$ in eq. (5) following from the Lagrangian (4),

$$
\left.\hat{\mathbf{n}} \cdot(\nabla \phi-e \mathbf{A})\right|_{\partial \Sigma}=0,
$$

in the dual variables become

$$
\hat{\mathbf{n}} \times\left(\nabla \chi_{\mathbf{A}}-e \nabla \Upsilon\right)=0 .
$$

These conditions can generally be taken to be the vanishing of both $\chi_{\mathrm{A}}$ and $\Upsilon$ on smooth boundaries because $\hat{\mathbf{n}} \times \nabla$ is the derivative along the boundary. The $\chi_{\mathrm{A}}$ for several vortices can then be superposed without changing the boundary conditions. 
The dual variables are useful for constructing the collective action for the charged vortices. According to the prescription (1), we substitute the ansatz (5) in the form of

$$
\psi=\sqrt{\rho_{0}} \exp \left(i \sum_{\mathrm{A}} n_{\mathrm{A}} \phi_{\mathrm{A}}\left(\mathbf{x}, \mathbf{X}^{\mathrm{A}}(t)\right)\right)
$$

into the action (4). In eq. (13) each $\phi_{\mathrm{A}}$ satisfies (7), $\rho_{0}$ is taken to be constant, and the total phase obeys boundary conditions (11). The result is the $U(1)$ sigma-model action

$$
L=\int d^{2} x\left(-\sum_{\mathrm{A}} \rho_{0} n_{\mathrm{A}} \dot{\phi}_{\mathrm{A}}-\alpha \sum_{\mathrm{A}, \mathrm{B}}\left(n_{\mathrm{A}} \nabla \phi_{\mathrm{A}}-e \mathbf{A}\right) \cdot\left(n_{\mathrm{B}} \nabla \phi_{\mathrm{B}}-e \mathbf{A}\right)\right) .
$$

Following Lund ${ }^{[4]}$ we put a fictitious extra boundary in the sample consisting of a small circle around each vortex and cuts $\mathcal{S}_{\mathrm{A}}$ from each vortex point $\mathbf{X}^{\mathrm{A}}$ to a fixed point $\mathbf{x}_{0}$ on the boundary. This has the effect of making every quantity in the integrand single-valued in the cut region. Each $\phi_{\mathrm{A}}$ jumps by $-2 \pi$ on its cut $\mathcal{S}_{\mathrm{A}}$. The action following from (14) reduces to

$$
\begin{aligned}
& S_{V}=\int d t\left[\sum_{\mathrm{A}} \pi \rho_{0} n_{\mathrm{A}} \epsilon_{a b} X_{a}^{\mathrm{A}} \dot{X}_{b}^{\mathrm{A}}-4 \pi \alpha \sum_{\mathrm{A}<\mathrm{B}} n_{\mathrm{A}} n_{\mathrm{B}} \int_{\mathcal{S}_{\mathrm{A}}} d \boldsymbol{\ell} \cdot \varepsilon \cdot \nabla \phi_{\mathrm{B}}\right. \\
&\left.-2 \pi \alpha \sum_{\mathrm{A}} n_{\mathrm{A}}^{2} \int_{\mathcal{S}_{\mathrm{A}}} d \boldsymbol{\ell} \cdot \varepsilon \cdot \nabla \phi_{\mathrm{A}}+4 \pi \alpha e \sum_{\mathrm{B}} n_{\mathrm{B}} \int_{\mathcal{S}_{\mathrm{B}}} d \boldsymbol{\ell} \cdot \varepsilon \cdot \mathbf{A}\right],
\end{aligned}
$$

where terms not depending on the vortex centers have been dropped and the cuts $\mathcal{S}_{\mathrm{A}}$ from the vortex $A$ to a fixed point on the boundary are explicitly

$$
\mathcal{S}_{\mathrm{A}}=\left\{\mathbf{x}(s, t) \mid 0 \leq s \leq 1, \quad \mathbf{x}(0, t)=\mathbf{X}^{\mathrm{A}}(t), \quad \mathbf{x}(1, t)=\mathbf{x}_{0} \in \partial \Sigma\right\}
$$

To obtain (15) we used the divergence theorem and the fact that the boundary conditions (3) ensure that there is no contribution from the physical boundary to the vortex action $S_{V}$. We also dropped as negligible the contributions from the small boundary circles around each vortex. The first term results from a double integration by parts. ${ }^{[4]}$ In the dualized variables 
the collective action becomes

$$
\begin{aligned}
S_{V}\left[\left\{\mathbf{X}^{\mathrm{A}}\right\}\right]=\int d t & \left\{\sum_{\mathrm{A}} \pi \rho_{0} n_{\mathrm{A}} \epsilon_{a b} X_{a}^{\mathrm{A}} \dot{X}_{b}^{\mathrm{A}}+4 \pi \alpha \sum_{\mathrm{A}<\mathrm{B}} n_{\mathrm{A}} n_{\mathrm{B}} \int_{\mathcal{S}_{\mathrm{A}}} d \boldsymbol{\ell} \cdot \nabla \chi_{\mathrm{B}}\right. \\
& \left.+2 \pi \alpha \sum_{\mathrm{A}} n_{\mathrm{A}}^{2} \int_{\mathcal{S}_{\mathrm{A}}} d \boldsymbol{\ell} \cdot \nabla \chi_{\mathrm{A}}-4 \pi \alpha e \sum_{\mathrm{B}} n_{\mathrm{B}} \int_{\mathcal{S}_{\mathrm{B}}} d \boldsymbol{\ell} \cdot \nabla \Upsilon\right\} .
\end{aligned}
$$

We observe that $\chi_{\mathrm{B}}(\mathbf{x})$ is the Green function $G_{D}\left(\mathbf{x}, \mathrm{X}^{\mathrm{B}}\right)$ satisfying zero Dirichlet boundary conditions. Because $\Upsilon$ satisfies $\nabla^{2} \Upsilon=-B(\mathbf{x}),\left.\Upsilon\right|_{\partial \Sigma}=0$, we also have $\Upsilon(\mathbf{x})=$ $\frac{1}{2 \pi} \int_{\Sigma} G_{D}\left(\mathbf{x}, \mathbf{x}^{\prime}\right) B\left(\mathbf{x}^{\prime}\right) d^{2} x^{\prime}$. The expression for the charged vortex collective action in a finite size region under the influence of an arbitrary external magnetic field can be written in terms of the Dirichlet Green function for the region as

$$
\begin{aligned}
S_{V}\left[\left\{\mathbf{X}^{\mathrm{A}}\right\}\right]=\int d t\{ & \sum_{\mathrm{A}} \pi \rho_{0} n_{\mathrm{A}} \epsilon_{a b} X_{a}^{\mathrm{A}} \dot{X}_{b}^{\mathrm{A}}-4 \pi \alpha \sum_{\mathrm{A}<\mathrm{B}} n_{\mathrm{A}} n_{\mathrm{B}} G_{D}\left(\mathbf{X}^{\mathrm{A}}, \mathbf{X}^{\mathrm{B}}\right) \\
& -2 \pi \alpha \sum_{\mathrm{A}} n_{\mathrm{A}}^{2} G_{D}^{\mathrm{reg}}\left(\mathbf{X}^{\mathrm{A}}, \mathbf{X}^{\mathrm{A}}\right) \\
& \left.+2 \alpha e \sum_{\mathrm{A}} n_{\mathrm{A}} \int_{\Sigma} d^{2} x G_{D}\left(\mathbf{X}^{\mathrm{A}}, \mathbf{x}\right) B(\mathbf{x})\right\} .
\end{aligned}
$$

Here we have ignored an infinite self-energy contribution to $S_{V}$ which does not depend upon the vortex centers by using the regularized Green function

$$
G_{D}^{\mathrm{reg}}(\mathbf{x}, \mathbf{x})=\lim _{\mathbf{y} \rightarrow \mathbf{x}}\left(G_{D}(\mathbf{x}, \mathbf{y})+\ln |\mathbf{x}-\mathbf{y}|\right)
$$

The vortex collective Hamiltonian is thus the same as the interaction energy for an assembly of charges, $q^{\mathrm{A}}=n_{\mathrm{A}}$, in the bounded region $\Sigma$ interacting with a background charge density proportional to the applied magnetic field, $B(\mathbf{x}) / 2 \pi$. The first term in the action, however, is not the usual kinetic term, so the motion of the charged vortices is quite different from that of point charges. In particular, the vortices do not experience an acceleration which is proportional to the gradient of the potential, but rather follow the instantaneous equipotentials described by the last three terms of (18) with speeds proportional to the gradient of the potential. A single vortex has as its orbits the equipotentials of a region $\Sigma$ with charge density $B(\mathbf{x}) / 2 \pi$, whose boundary, $\partial \Sigma$, is held at constant potential. The fact 
that the phase space of the vortices is just the two-dimensional sample itself together with the identification of the vortex Hamiltonian as an electrostatic energy illuminates the plasma analogy of the quantum Hall effect. If the density matrix computed from the Laughlin wave function is identified with the density matrix of a canonical ensemble of vortices, we have immediately

$$
\left|\Psi_{\text {Laughlin }}\right|^{2}=\varrho_{\text {Laughlin }}=\varrho_{V}=\exp \left(-\beta H_{V}\right)
$$

\section{ACKNOWLEDGEMENTS}

It is a pleasure to thank L. Durand for a useful conversation which motivated this investigation, and R.P. Springer for helpful comments on the manuscript. This work was supported in part by DOE grant No. DE-AC02-76-ER00881.

\section{REFERENCES}

1. S.B. Giddings and F. Wilczek, Mod. Phys. Lett. A5 (1990) 635.

2. S. Girvin and A. MacDonald, Phys. Rev. Lett. 58 (1987) 1252;

S.M. Girvin, in The Quantum Hall Effect, R.E. Prange and S.M. Girvin, eds., (Springer Verlag, New York, 1987) p. 389;

S.C. Zhang, T.H. Hansson and S. Kivelson, Phys. Rev. Lett. 62 (1989) 82;

N. Read, Phys. Rev. Lett. 62 (1989) 86.

3. T.J. Allen and A.J. Bordner, Charged Vortex Dynamics in Ginzburg-Landau Theory of the Fractional Quantum Hall Effect, UW-Madison preprint MAD/TH-92-02, hepth/9206073.

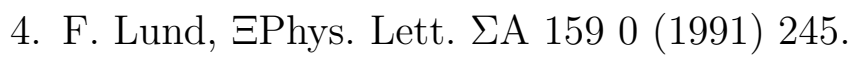

